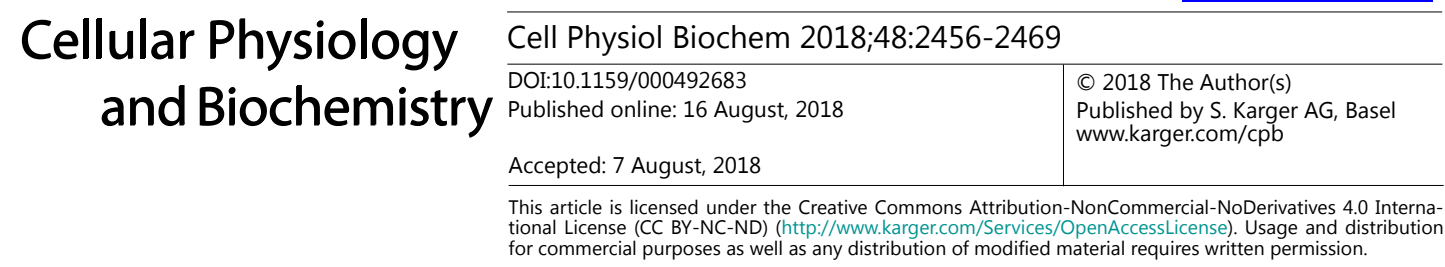

\title{
High Platelet Levels Attenuate the Efficacy of Platinum-Based Treatment in Non-Small Cell Lung Cancer
}

\author{
Zhangfei Wang ${ }^{\mathrm{a}}$ Man Fang ${ }^{\mathrm{a}}$ Juan Li ${ }^{\mathrm{b}}$ Ruining Yang ${ }^{\mathrm{c}}$ Jianping Du ${ }^{\mathrm{d}}$ Yiqin Luo \\ aDepartment of Laboratory Medicine, the Affiliated Anhui Provincial Hospital of Anhui Medical \\ University, Hefei, b ${ }^{b}$ epartment of Blood Transfusion, the Affiliated Anhui Provincial Hospital of Anhui \\ Medical University, Hefei, 'Department of Laboratory Medicine, the People's Liberation Army 81 \\ Hospital, Nanjing, 'The Center of Tumor Therapy, the Affiliated Anhui Provincial Hospital of Anhui \\ Medical University, Hefei, China
}

\section{Key Words}

Platelets • Cisplatin • Apoptosis • Lung cancer • Chemotherapy response

\begin{abstract}
Background/Aims: The correlation between platelet levels and clinical outcomes has received increasing attention, but it is not yet clear whether and how platelet levels affect the therapeutic response in non-small cell lung cancer (NSCLC). In the current study, we aimed to explore the role of platelet levels in responsive to platinum-based chemotherapy and investigated the underlying mechanism. Methods: We evaluated the possibility of platelet level as a biomarker for response to platinum-based therapy in NSCLC by retrospective analysis of NSCLC patients. Cell proliferation was evaluated using cell counter and flow cytometry. Cell capillary-like structures of HPMEC were estimated with ECMatrix. The effect of platelets on A549, H1299, and HPMEC apoptosis was measured by flow cytometry. A A549-bearing NOD/ SCID mice model was employed to determine whether platelets could counteract cisplatininduced apoptosis in vivo. In vivo cell proliferation and apoptosis were evaluated with Ki-67 antibody and TUNEL staining respectively. The angiogenesis of tumor was estimated by CD31 microvessel density. The protein levels of Akt, Bad and $\mathrm{Bcl}-2$ were assessed by western blot. To further examine platelet-driven effects of the chemotherapeutic response, we used platelet depletion and platelet transfusion in A549-bearing NOD/SCID mice. Results: Thrombocytosis at NSCLC diagnosis was associated with lower progression-free survival and median overall survival. Platelet levels before chemotherapy in the no response group were markedly higher than in the responsive group. Platelets rescued the inhibition of cell proliferation and angiogenesis and protected against cell apoptosis induced by cisplatin, platelets rescued cisplatin-induced apoptosis via the $\mathrm{Akt} / \mathrm{Bad} / \mathrm{BCl}-2$ signaling pathway under endoplasmic reticulum stress. Platelet transfusion decreased the therapeutic effect of cisplatin, while it
\end{abstract}

Z. Wang and M. Fang contributed equally to this work.

\begin{tabular}{ll}
\hline Yiqin Luo & Department of laboratory medicine, The Affiliated Anhui Provincial Hospital of Anhui Medical University \\
& No17, Lujiang Rd, Hefei 23001 (China) \\
& Tel. +86-551-62283975, Fax +86-551-62283975, E-Mail luoyiqin2004@hotmail.com
\end{tabular}


Wang et al.: Platelet Levels Affect Response to Platinum in NSCLC

was increased by platelet depletion. Conclusion: We confirmed an important anti-apoptosis mechanism mediated by platelets and found that platelets could counteract cisplatin-induced apoptosis. Reducing platelet levels or blocking platelet-based cytoprotection may represent new methods for improving the chemotherapeutic effect.

(C) 2018 The Author(s)

Published by S. Karger AG, Basel

\section{Introduction}

Platelets have an important function in blood coagulation. However, they are also involved in many non-hemostatic functions, including tissue repair [1] , inflammation [2], lymphatic development, and angiogenesis [3]. Platelets also boost cancer metastasis and cell proliferation by pericellular adhesion and signal transduction [4-6]. Such pleiotropic effects are possible due to the numerous molecules released from activated platelets. It has been reported that thrombocytosis occurs in association with solid tumors and over $40 \%$ of patients with thrombocytosis have occult metastasis. Platelets have been shown to mediate the escape of micrometastases from NK cells [7]. Direct signaling between cancer cells and platelets leads to epithelial-to-mesenchymal transition [8]. High platelet counts have been confirmed to correlate with a poor prognosis in many distinct tumor entities, including ovarian cancer [9], gastric cancer [10], hepatocellular carcinoma [11], and lung cancer [12]. Colonic adenocarcinoma cells exposed to platelets have enhanced survival and in vitro paclitaxel and 5-FU chemoresistance. Cancer cells incubated with platelets have downregulated pro-apoptotic and up-regulated anti-apoptotic genes, fewer cells in the quiescent phase, an increased number of cells in DNA synthesis, enhanced DNA repair proteins, and express cyclins and MAPKs [6]. Results from a study of patients with ovarian cancer also showed that increased platelet levels are associated with higher rates of recrudescence and lower rates of response to taxane chemotherapy [13].

Lung cancer is the main cause of cancer deaths worldwide, largely due to metastasis and development of resistance to treatment. Non-small cell lung cancer (NSCLC) is the most common type of lung cancer [https://www.cancerorg/content/dam/cancer-org/ research/cancer-facts-and-statistics/annual-cancer-facts-and-Fig.s/2016/cancer-factsand-Fig.s-2016.pdf]. However, it is not clear whether and how platelet levels affect treatment response in NSCLC. In the present study, we explored the effect of platelet counts on response to platinum-based chemotherapy and the underlying mechanisms using clinical data, in vitro cells, and an in vivo mouse model.

\section{Materials and Methods}

\section{Subjects}

Patients at our hospital with cytologically or histologically verified NSCLC ( $\mathrm{n}=167$ ) between September 2006 and July 2013 were eventually included in the study. Initially, 334 NSCLC patients (126 women and 208 men), with an average age of 57 (range 30-74) years were screened. The exclusion criteria were as follows: patients with disorders known to be relevant to reactive thrombocytosis, or concomitant myeloproliferative conditions such as autoimmune disorders, inflammatory diseases, and other advanced cancers; patients without known platelet levels; patients who did not accept initial treatment or followup at the study hospital. Full medical records were retrospectively reviewed to gain the following patient parameters: age, sex, platelet levels, tumor differentiation, TNM stage, and cell type. Among these patients, 186 received platinum-based chemotherapy. Platelet levels were recorded at the time of the initial assessments, throughout treatment, after chemotherapy, and during the post-treatment monitoring period. In our laboratory, the $95 \%$ confidence limit (normal range) of platelet levels is considered to be 100-300 $\times 10^{9} / \mathrm{L}$. Thrombocytosis was considered when platelet levels were $>400 \times 10^{9} / \mathrm{L}$. The follow-up time was 60 months. The survival time of patients was calculated from the date of chemotherapy to the date of death. Progression-free survival and overall survival were determined from the start of therapy to the first recorded progression of disease and death, respectively. The study plan and the contact materials were 


\section{Cellular Physiology Cell Physiol Biochem 2018;48:2456-2469 \begin{tabular}{l|l|l} 
DOI: 10.1159/000492683 & $\begin{array}{l}\text { O 2018 The Author(s). Published by S. Karger AG, Basel } \\
\text { www.karger.com/cpb }\end{array}$
\end{tabular}}

Wang et al.: Platelet Levels Affect Response to Platinum in NSCLC

checked and approved by the Institutional Review Board of Anhui Provincial Hospital (Hefei, China). The entire study adhered to the principles and guidelines of Helsinki Declaration, and informed written consent was obtained from all qualifying patients. Moreover, the comparator group, that is, 300 patients with chronic obstructive pulmonary disease or bronchial asthma (60 women and 100 men) with an average age of 50 (range 28-65) years, were recruited by the Medical Examination Center of the Anhui Provincial Hospital during the period mentioned above. None of the patients in the comparator group had malignant tumors.

\section{Chemotherapy regiment}

Cisplatin plus gemcitabine (PG planning) was administered to all qualified patients. Patients received cisplatin $\left(75 \mathrm{mg} / \mathrm{m}^{2}\right)$ on days $1-3$ and gemcitabine hydrochloride $\left(1250 \mathrm{mg} / \mathrm{m}^{2}\right)$ on days 1 and 8 . Drugs were administered on a 21-day cycle for two therapy courses in total.

\section{Evaluation of the therapeutic effect}

One week after chemotherapy, all patients underwent a further pulmonary computed tomography (CT) scan. The therapeutic effect was evaluated based on Response Evaluation Criteria In Solid Tumors (RECIST) [14]: (1) complete response (CR), all detectable symptoms faded away; (2) partial response (PR), $\geq 30 \%$ shrinkage in the sum of linear tumor measurements; (3) progressive disease (PD), new lesions faded away and $\geq 20 \%$ increase in the sum of target lesions; and (4) stable disease (SD); neither enough of a decrease for PR nor enough of an increase for PD. CR and PR were considered effective responses, and SD and PD were considered ineffective responses.

\section{Platelet isolation}

Platelets were isolated from anticoagulated blood (sodium citrate, 1:9 vol/vol) drawn from healthy subjects by venipuncture. Briefly, anticoagulated blood was centrifuged at $200 \times g$ for 20 min to get plateletrich plasma (PRP). PRP was again centrifuged at $1000 \times g$ for $10 \mathrm{~min}$ in the presence of prostacyclin $(0.1$ $\mu \mathrm{g} / \mathrm{mL}$ ). The platelet pellet was resuspended and washed twice in modified HEPES/Tyrode buffer with prostacyclin. Purified platelets were resuspended in the same buffer at the required concentrations. The platelets were pooled and then divided into aliquots. Informed written consent was obtained from all healthy subjects.

\section{Cell lines and culture}

Human pulmonary microvascular endothelial cells (HPMECs) and human lung cancer A549 and H1299 cell lines (cisplatin-sensitive cell) were obtained from the Cell Bank of the Chinese Science Academy (Shanghai, China). The cell lines were cultured in RPMI-1640 medium containing $10 \%$ fetal bovine serum in $5 \% \mathrm{CO}_{2}$ and $95 \%$ air at $37^{\circ} \mathrm{C}$. Subconfluent cells were then inoculated into $75-\mathrm{mm}^{2}$ flasks and cultured in serum-free medium 1 day before treatment.

\section{Antibodies and reagents}

The antibodies used were anti-cleaved-caspase-3, anti-basic fibroblast growth factor (bFGF), anti-transforming growth factor $\beta$ (TGF- $\beta$ ), anti- $\beta$-actin, anti- $\beta$-tubulin, anti-CHOP, anti-Bad, anti-Bcl-2, phosphorylated Bad, anti-Erk1/2, phosphorylated Erk1/2 (p-Erk1/2), anti-Akt, and phosphorylated Akt (p-Akt) and purchased from Cell Signaling Technology (Danvers, MA). The phosphorylated protein kinase R-like endoplasmic reticulum kinase (p-PERK) antibodies and anti-protein kinase R-like endoplasmic reticulum kinase (PERK) were purchased from Santa Cruz Biotechnology (Santa Cruz, CA). The plateletdepleting antibody used was a rat anti-mouse CD42b/GPIb $\alpha$ mAb (GTI Diagnostics GmbH, Aachen, Germany). GSK2656157, TCN, and SC79 were purchased from Selleck (Shanghai, China). GSK 2656157 is a specific inhibitor of PERK. SC79 is a unique specific activator of Akt, and TCN is a selective AKT inhibitor.

\section{In vitro assays}

Cell proliferation was evaluated using Cell Counting Kit 8 (CCK-8; Sigma-Aldrich, Shanghai, China). A total of $5 \times 10^{3}$ cells per well were inoculated on culture plates and grown for the indicated times. A group that contained no cells served as the control. CCK-8 solution $(10 \mu \mathrm{L})$ was added into each well of the plates and cultured at $37^{\circ} \mathrm{C}$ for $1 \mathrm{~h}$. The absorbance was measured at a wavelength of $450 \mathrm{~nm}$ to determine cell proliferation.

\section{KARGER}




\section{Cellular Physiology Cell Physiol Biochem 2018;48:2456-2469 \begin{tabular}{l|l|l} 
and Biochemistry Published online: 16 August, 2018 & $\begin{array}{l}\text { @ } 2018 \text { The Author(s). Published by S. Karger AG, Basel } \\
\text { www.karger.com/cpb }\end{array}$ \\
\hline
\end{tabular}}

An EdU-488 cell proliferation kit (Promega, Shanghai, China) was applied and the results were assessed by flow cytometry. Approximately $2 \times 10^{4}$ cells per well were seeded onto the culture plates and cultured at $37^{\circ} \mathrm{C}$ for $12 \mathrm{~h}$. A mixture of EdU working solution, containing $0.20 \mu \mathrm{L}$ EdU and $100 \mu \mathrm{L}$ complete culture medium, was added to each well of the plates. After incubation at $37^{\circ} \mathrm{C}$ for $3 \mathrm{~h}$, trypsin-EDTA was added and cells were collected, rinsed twice with phosphate-buffered saline (PBS), and fixed in 4\% paraformaldehyde for $10 \mathrm{~min}$. The fixed cells were neutralized with glycine $(3 \mathrm{mg} / \mathrm{mL})$ and rinsed twice. Cells were permeabilized with $0.5 \%$ Triton X-100, labeled with Apollo staining (Guangzhou RiboBio Co., Ltd., Guangzhou, China), and the prepared cells were analyzed by flow cytometry.

The tube formation assay was performed as described in the manufacturer's instructions (Collaborative Biomolecules, Bedford, MA). To study the tube-like construction activity of the HPMECs, capillary-like structures were estimated with ECMatrix. $(60 \mu \mathrm{L})$, which was added into each well of the culture plate. After pretreatment with culture liquid in the absence or presence of platelets $\left(4 \times 10^{7}\right)$ and cisplatin for $48 \mathrm{~h}, 5 \times$ $10^{3} \mathrm{HPMECs}$ in $80 \mu \mathrm{L}$ culture liquid were inoculated onto the ECMatrix for $25 \mathrm{~min}$. After $8 \mathrm{~h}$, vessel formation was determined under a light microscope.

The Annexin V-FITC apoptosis test kit (BioVision Inc., Milpitas, CA) was applied to evaluate the effect of platelets on A549, H1299, and HPMEC apoptosis following the instructions. Briefly, after $24 \mathrm{~h}$ of inoculation of platelets and/or cisplatin into serum-free culture liquid, all cells were collected, rinsed with Dulbecco's phosphate-buffered saline, and resuspended in $100 \mu \mathrm{L}$ of binding buffer. Cells were cultured with Annexin V-FITC and propidium iodide (PI). Finally, the mixture was diluted to volume of $250 \mu \mathrm{L}$ and analyzed by flow cytometry.

\section{Plasmid construction and cell transfection}

The Bcl-2 expression plasmid was amplified by PCR, enzyme digested, and the product was purified by PCR. Bcl-2 cDNA was cloned into a pEGF vector (Promega Corp., Beijing, China) to obtain pEGF-C3-Bcl-2. For the Bad silenced plasmid, the annealed Bad shRNAs were cloned into pSUPER vector (Promega Corp.) to obtain pSUPER-shBad. The plasmids containing target genes were confirmed by sequencing. In accordance with the manuals, stable transfected Bcl-2 and shBad cell lines were established via retrovirus infection and antibiotic selection

\section{Western blots}

Western blots were executed as described previously [15]. Briefly, after cells were lysed, proteins were subjected to sodium dodecyl sulfate polyacrylamide gel electrophoresis, transferred to nitrocellulose Hybond C membrane, blocked in blocking buffer (5\% w/v non-fat milk and $0.5 \%$ Tween 20 ) and detected with antibodies. The immunoreactive proteins were detected by a chemiluminescence reagent (Tiangen Biochem, Beijing, China).

\section{In vivo experiments}

All studies involving animals were approved by the Animal Care and Treatment Committee of Anhui Provincial Hospital. Female NOD/SCID mice were obtained from the Experimental Animal Centre of the institution. A549 and H1299 cells were used for the animal NOD/SCID models. A549 or H1299 cells (2 × $10^{6}$ ) were resuspended in $200 \mathrm{~mL}$ of serum-free Dulbecco's modified Eagle's medium culture liquid. NOD/ SCID mice (6-8 weeks old) were injected subcutaneously. Tumor volume was determined using electronic calipers and tumor size was measured using the following calculation: tumor volume $=\left(\alpha^{2} \times b\right) / 2$, where $\alpha$ is the width and $b$ is the length of the tumor. When the average tumor volume reached $90-100 \mathrm{~mm}^{3}$ in size, the mice were randomly divided into three groups ( $n=5$ mice/group): (a) control (200 mL PBS three times weekly by tail vein injection); (b) cisplatin alone ( $5 \mathrm{mg} / \mathrm{kg}$ three times weekly by intraperitoneal injection); and (c) cisplatin/platelet transfusion combination $(5 \mathrm{mg} / \mathrm{kg}$ cisplatin three times weekly by intraperitoneal injection and platelet transfusion $\left[2 \times 10^{8}\right]$ three times weekly by tail vein injection). To explore the effect of chemotherapy on thrombocytopenia or on thrombocytosis in vivo, A549 and H1299 cells were injected into NOD/SCID mice. When the average tumor volume reached about $100 \mathrm{~mm}^{3}$ in size, mice were randomly divided into the following groups ( $\mathrm{n}=10 \mathrm{mice} /$ group): control group (IgG $0.5 \mu \mathrm{g} / \mathrm{g}$, tail vein injections three times weekly; control IgG plus cisplatin (control IgG $(0.5 \mu \mathrm{g} / \mathrm{g}$ via tail vein injection three times weekly and cisplatin $5 \mathrm{mg} / \mathrm{kg}$ i.p three times weekly); platelet depletion (0.5 $\mu \mathrm{g} / \mathrm{g}$ platelet-depleting antibody via 


\section{Cellular Physiology Cell Physiol Biochem 2018;48:2456-2469 \begin{tabular}{ll|l} 
and Biochemistry Published online: 16 August, 2018 & $\begin{array}{l}\text { (c) } 2018 \text { The Author(s). Published by S. Karger AG, Basel } \\
\text { www.karger.com/cpb }\end{array}$
\end{tabular}}

Wang et al.: Platelet Levels Affect Response to Platinum in NSCLC

tail vein injection three times weekly); cisplatin plus platelet depletion (cisplatin plus platelet-depleting antibody); platelet transfusion (tail vein transfusion of PRP isolated from NOD/SCID mice); and cisplatin plus platelet transfusion.

\section{Immunohistochemical staining}

Terminal deoxynucleotidyl transferase dUTP nick end labeling (TUNEL) staining was executed using an in situ Cell Death Detection Kit (Roche, Beijing, China) according to the recommended manual [16]. Sections of tumor tissue were incubated with $50 \mu \mathrm{L}$ TUNEL reaction mixture containing dUTP and TdT. The sections were then incubated in a dark humidified atmosphere for $1 \mathrm{~h}$ at $37^{\circ} \mathrm{C}$. Sections were washed three times with PBS (each wash lasted $10 \mathrm{~min}$ ) and visualized under a fluorescence microscope (emission wavelength: 515-565 nm; excitation wavelength: 450-500 $\mathrm{nm}$ ).

Immunohistochemical staining of cell proliferation was conducted on tumor sections. The first antibody was rat anti-mouse Ki-67 antibody (Santa Cruz Biotechnology) and the second was FITC-conjugated rabbit anti-rat IgG (Eton Bioscience, Inc., San Diego, CA). A total of 20 fields from three tumor tissues for each treatment group were counted and examined at a magnification of $400 \times$. Results were reported as percentage of Ki-67 positive cells \pm standard deviation per $\times 400$ magnification. The Ki- 67 proliferation index was found using the following calculation: (Ki-67-positive cell count)/(total number of cells) $\times 100 \%$.

Assessment of the microvessel density of tumors was performed as follows. Briefly, fixed and dried tumor sections were incubated with rat monoclonal anti-CD31 antibody (BD Biosciences. Franklin Lakes, $\mathrm{NJ}$ ) for $18 \mathrm{~h}$ at $4^{\circ} \mathrm{C}$ and then reacted for $1 \mathrm{~h}$ with goat anti-rat immunoglobulin G conjugated to Texas red (Vector Laboratories, Burlingame, CA) in the dark at room temperature. The CD31-positive vessel-like structures were counted in 5-10 random microscopic fields per section at a magnification of 200×.

\section{Statistical analysis}

Statistical analysis was conducted using GraphPad Prism 6 and Excel. Kaplan-Meier survival curves were evaluated by a two-sided log-rank test. All experiments were performed three times. The results are expressed as the means \pm standard error of the mean. Differences between the groups were assessed using the Student's t-test or one-way analysis of variance followed by a Tukey's multiple comparison post hoc test. A $p$-value $<0.05$ was considered statistically significant for all statistical analyses.

\section{Results}

Platelet levels as prognostic markers in NSCLC

We identified only 44 patients (13.17\%) with thrombocytosis at the time of their admission to hospital. The prevalence of thrombocytosis was $13.08 \%(28 / 214)$ in men and $13.3 \%(16 / 120)$ in women. Thrombocytosis was found in $4.05 \%(6 / 148)$ of stage I/ II patients and $20.43 \%(38 / 186)$ of stage III/IV patients. Platelet counts differed noticeably between the comparator and NSCLC groups before chemotherapy, with higher levels in the NSCLC group compared with the comparator group $(296.4 \pm 120.3$ vs. $167.5 \pm 80.3, t=$ 26.48, $p<0.001$ ). Kaplan-Meier survival analysis indicated that the progressionfree survival and overall survival times of the NSCLC patients with platelet levels at diagnosis of $<400 \times 10^{9} / \mathrm{L}$ were significantly longer than those of NSCLC patients with platelet levels at diagnosis of $\geq 400 \times 10^{9} / \mathrm{L}(p=0.04$ and $p=0.003$, respectively) (Fig. 1).

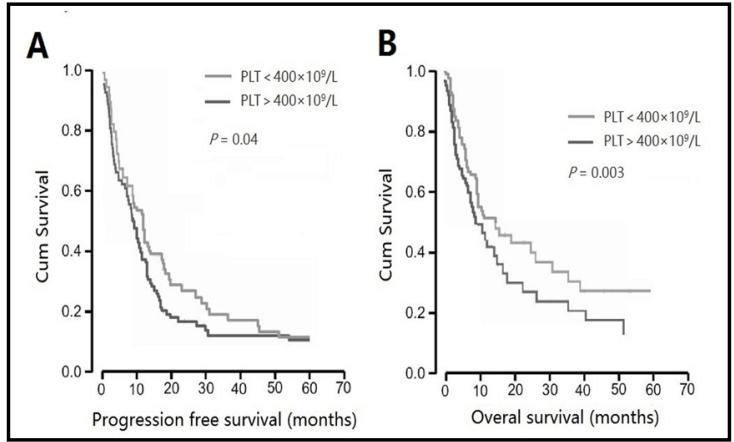

Fig. 1. Kaplan-Meier curves of survival in NSCLC patients. (A) Progression-free survival and (B) overall survival for those with thrombocytosis $\left(>400 \times 10^{9} / \mathrm{L}\right)$ and those with normal platelet counts $\left(<400 \times 10^{9} / \mathrm{L}\right)$. P values were measured by the log-rank test. 
Thrombocytosis is associated with resistance to chemotherapy

In order to test whether platelet counts could be biomarkers for early evaluations of chemotherapeutic response, we determined the platelet levels before and after chemotherapy. Platelet levels after chemotherapy steadily increased in 34 of 44 patients who had thrombocytosis before chemotherapy, whereas the levels of the remaining 10 patients had returned to within normal range within one month of chemotherapy. There was a trend towards an association between normalization of platelet levels after chemotherapy and patient survival, but we could not examine this statistically because the number of patients was too small.

Patients in III/IV stage $(n=186)$ received platinum-based chemotherapy. According to the RECIST guidelines and CT images, treatment in 72 NSCLC cases were considered as ineffective and 114 cases as effective. Clear differences were observed in platelet levels between the SD plus PD group and the CR plus PR group before chemotherapy $(p<0.05)$. In the CR plus PR group, the platelet levels were $247.6 \pm 89.6 \times 10^{9}$ before chemotherapy and 136.2 $\pm 95.6 \times 10^{9}$ after chemotherapy, indicating an obvious drop after chemotherapy $(p<0.01, t$ $=15.51)$. In contrast, a sharp increase in platelet levels was observed after chemotherapy in the SD plus PD group (355.2 $\pm 119.3 \times 10^{9}$ vs. $473.8 \pm 123.7 \times 10^{9}, p<0.01, t=22.38$ ) (Table 1 ). These results pointed to an association between the normalization of platelet levels during chemotherapy and disease response to treatment.

Effects of platelets on cell proliferation in cells exposed to cisplatin in vitro and in vivo

To investigate the effects of platelets on the proliferation of cells exposed to high or normal concentrations of cisplatin, A549 cells, H1299 cells, and HPMECs were incubated in conditioned culture containing cisplatin with or without platelets. CCK-8 analysis and EdU assay showed proliferation of all three type of cells (Fig. 2A, B). Cisplatin significantly reduced cell proliferation, whereas incubation with platelets resulted in a noticeable increase in the proliferation of all cell types. Furthermore, the cisplatinproduced inhibition of proliferation was markedly relieved by platelets in all three cell types.

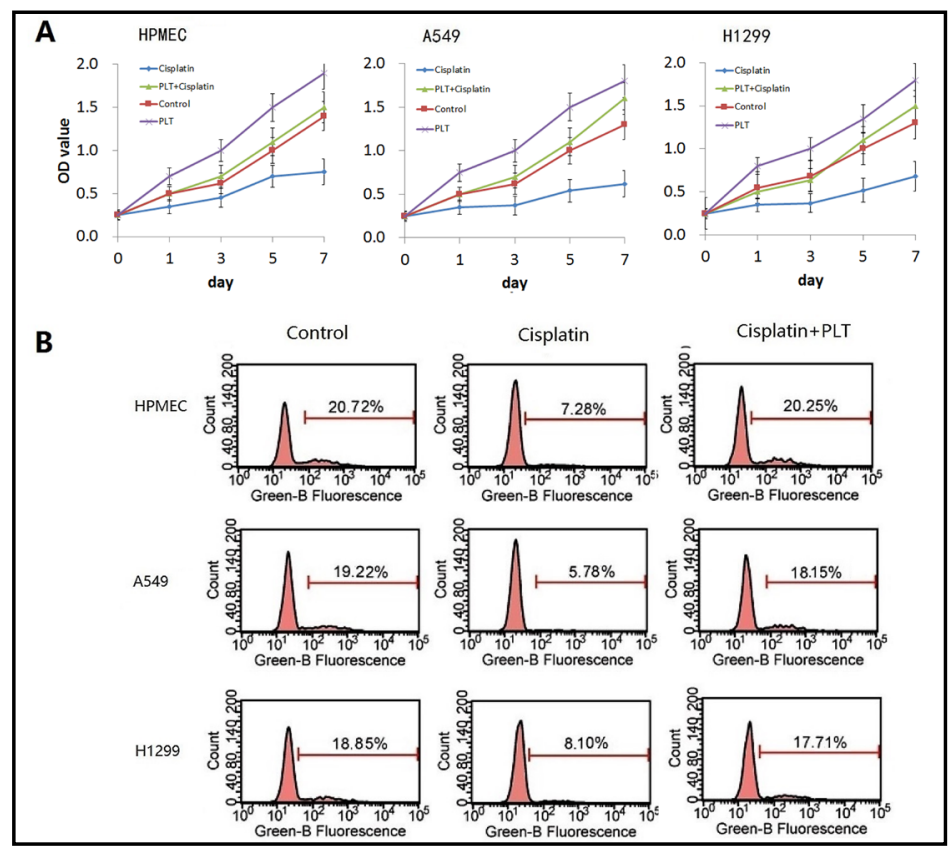

Fig. 2. Effects of PLT on cell proliferation in vitro and in vivo. (A) The proliferation of HPMEC, A549 and H1299 cells exposed to cisplatin with or without PLT, tested by CCK-8 kit $(n=3)$. (B) The viability of HPMEC, A549 and H1299 cells exposed to cisplatin with or without PLT was verified by EdU kit $(n=3)$. Results are showed as mean $+/-S D$. $* \mathrm{p}>0.05, * * \mathrm{p}<0.05$.
Table 1. The changes of PLT level in patients with NSCLC before and after chemotherapy ( $x \pm s)$. CR complete response, PR partial response, PD progressive disease, SD stable disease

\begin{tabular}{|c|c|c|c|c|c|}
\hline Effic: & Number & PLT level $(x$ & & $t$ & $p$ \\
\hline & & Before chemotherapy & After chemotherapy & & \\
\hline $\mathrm{CR}+\mathrm{PR}$ & 114 & $247.6 \pm 89.6$ & $136.2 \pm 95.6$ & 13.7 & $<0.01$ \\
\hline $\mathrm{SD}+\mathrm{PD}$ & 72 & $355.2 \pm 119.3$ & $473.8 \pm 123.7$ & 22.30 & $<0.01$ \\
\hline
\end{tabular}


Effects of platelets on vessel-like structures of cells or angiogenesis in tumor tissue exposed to cisplatin in vitro and in vivo

The tube formation assay showed that cisplatin had strong anti-angiogenic activity; no vessel-like structures formed-not even slightly-after only $12 \mathrm{~h}$ of treatment (Fig. 3A).

Western blots indicated high expression of vascular endothelial growth factor (VEGF), platelet-derived growth factor (PDGF) BB, and bFGF in the platelet group (Fig. 3B); thus, Erk and Akt signals would be easily activated. To confirm this speculation and explore whether these signaling molecules respond to the action of platelets, western blotting was used to detect the expression of p-Erk1/2, Erk1/2, p-Akt, and Akt in HPMECs with or without cisplatin plus platelets. Compared with cisplatin, HPMECs incubated with platelets showed an obvious increase in the phosphorylation levels of Erk and Akt, whereas the phosphorylation levels in the control were unchanged, suggesting that platelets can protect angiogenesis in the presence of cisplatin (Fig. 3C).

Western blotting showed that the expression of VEGF-A in HPMECs exposed to cisplatin was lower than that in the control. Nevertheless, VEGF-A expression in cells exposed to cisplatin plus platelets was significantly higher than in cells exposed to cisplatin alone (Fig. 3D).

Cisplatin reduced the number of tumor endothelial cells. To confirm the suppression of tumor angiogenesis, CD31 staining indicated that the number of endothelial cells in tumor tissues was greatly reduced in the cisplatin-treated group compared with the other treatment groups, (Fig. 3A, top), suggesting that cisplatin significantly restricted the blood supply in tumor tissues but that co-treatment with platelets had a shielding effect on angiogenesis (Fig. 3E, F).

Effects of platelets on cell apoptosis induced by cisplatin in vitro and in vivo

Annexin V-FITC/PI double staining revealed cells in early apoptosis in vitro. The data indicated that exposure to cisplatin significantly increased apoptosis compared to the control, while platelets alleviated this effect in A549 cells, H1299 cells, and HPMECs. Moreover, A549 cells, H1299 cells, and HPMECs treated with platelets alone showed fewer apoptotic cells than the control (Fig. 4A-C). Western blotting showed that after $48 \mathrm{~h}$ of exposure to

Fig. 3. Effects of PLT on angiogenesis exposed to cisplatin in vitro and in vivo. (A) The angiogenesis of HPMEC cells exposed to cisplatin with or without PLT was assessed by vessel formation kit $(\mathrm{n}=3)$. (B) VEGF, PDGFBB and bFGF were highly expressed in the PLT. (C) After cell exposure to cisplatin with or without PLT for 3h, western blotting showed the expression of Erk, p-Erk, Akt and p-Akt. (D) After cell exposure to cisplatin with or without PLT for $24 \mathrm{~h}$, western blotting showed the expression of VEGF-A. (E, F) CD31 immunostaining showed the effects of PLT on angiogenesis in tumor tissue ( $\mathrm{n}=5$ mice/group). Scale bar: (A, E) $100 \mu \mathrm{m}$. Results are showed as mean + -SD. $* \mathrm{p}>0.05, * * * \mathrm{p}<0.01$.

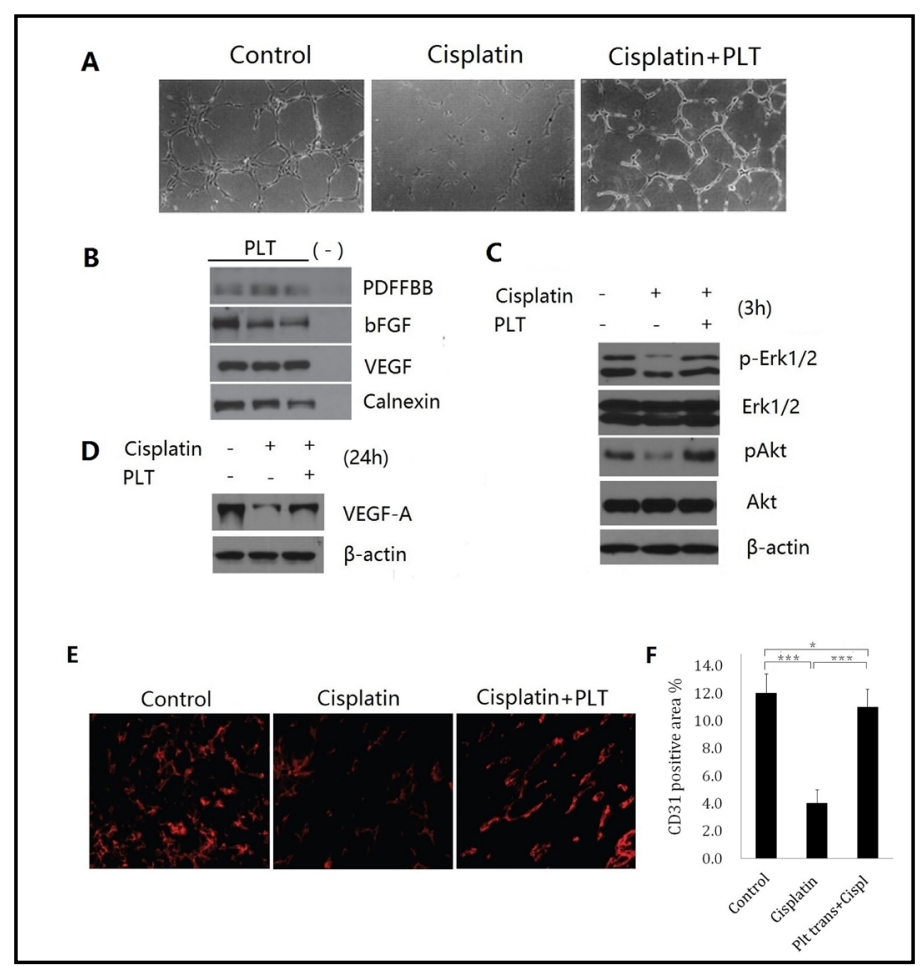




\section{Cellular Physiology Cell Physiol Biochem 2018;48:2456-2469 and Biochemistry \begin{tabular}{l|l} 
DOI: 10.1159/000492683 & $\begin{array}{l}\text { C } 2018 \text { The Author(s). Published by S. Karger AG, Basel } \\
\text { www.karger.com/cpb }\end{array}$
\end{tabular}

cisplatin, caspase-3 was activated, but it was inhibited by co-treatment with platelets in A549, H1299, and HPMECs (Fig. 4D).

The in vivo anti-apoptosis effect of platelets in the A549 mouse model were assessed by TUNEL analysis. The results showed that the number of apoptotic cells in the cisplatin group increased compared with the control group, while that in the cisplatin plus platelet group was reduced by platelet transfusions (Fig. 4E, F), suggesting that platelets could promote A549 lung cancer by counteracting apoptosis and thereby preventing the development of necrosis in the tumor tissue.

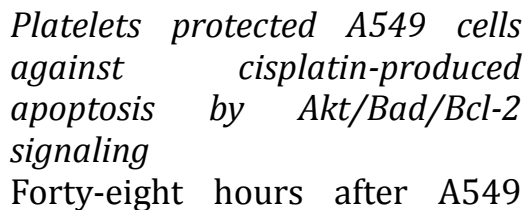
Forty-eight hours after A549
were exposed to platelets or cells were exposed to platelets or
cisplatin, western blotting indicated that high-concentrations of cisplatininduced endoplasmic reticulum stress (ERS) and activated PERK. CHOP (CCAAT/enhancer binding proteins), a downstream molecule of PERK [17], inhibits the expression of $\mathrm{Bcl}-2$, which is an anti-apoptotic protein, and activates the caspase cascade reaction, resulting in cell apoptosis [18].

The key pathway of ERS-induced apoptosis is PERK signaling. When A549 cells were exposed to PERK inhibitor GSK2656157 (15 nM) plus cisplatin, the expression of p-PERK and CHOP dropped, Bcl-2 expression was up-regulated, and cleavedcaspase-3 was not detected (Fig. 5A). To further determine the role of Bcl-2, we over-expressed it in A549 cells by $b c l-2$ transfection. Cisplatin still induced the phosphorylation of PERK and up-regulation of CHOP, but CHOP inhibition of Bcl-2 was impeded and caspase- 3 was not activated. When A549 cells were exposed to platelets plus cisplatin, platelets reduced the inhibition of Bcl-2, and caspase- 3 was not activated. The data indicated that platelets relieved the ERS-created apoptosis by maintaining the expression of Bcl-2 without changing PERK activation and CHOP expression (Fig. 5A). Therefore, in order to study the inhibitory mechanism of platelets in cisplatin-created apoptosis, A549 cells were exposed to platelets plus cisplatin. Western blots showed that Akt and Bad were phosphorylated, Bcl-2 levels were increased, and cleaved-

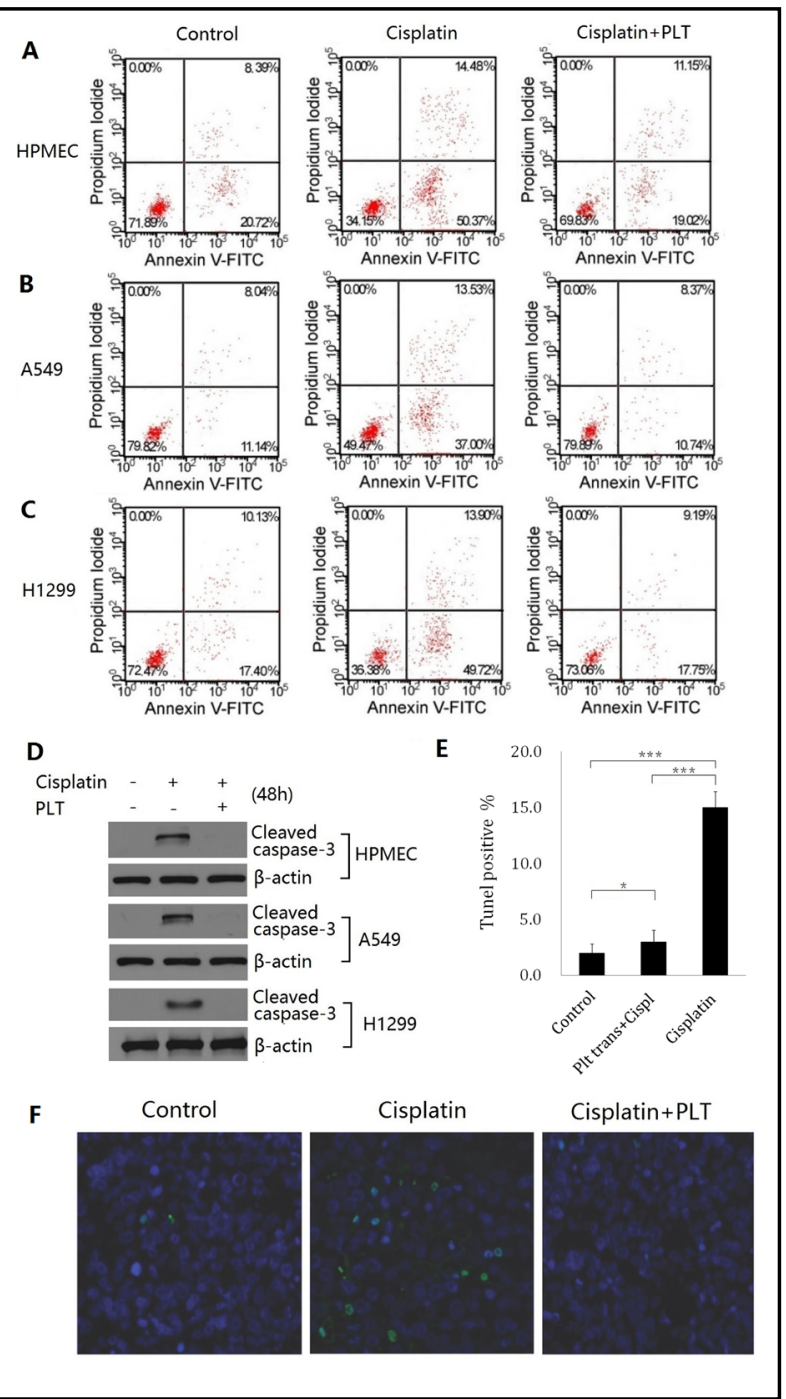

Fig. 4. Effects of PLT on cisplatin-produced cell apoptosis in vitro and in vivo. (A-C) Annexin V-FITC/PI double staining showed the apoptosis of HPMEC, A549 and H1299 cells exposure to cisplatin with or without PLT $(n=3)$. (D) Western blotting showed the cleaved caspase-3 in HMPEC, A549 and H1299 cells exposed to cisplatin with or without PLT. (E, F)TUNEL analysis showed the anti-apoptosis effects of PLT on the A549 xenograft mouse models. Representative images from tumors of at least 5 mice per group. Scale bar: (F) $100 \mu \mathrm{m}$. Results are showed as mean $+/$-SD. *p $>0.05$, * $* * \mathrm{p}<0.01$. 


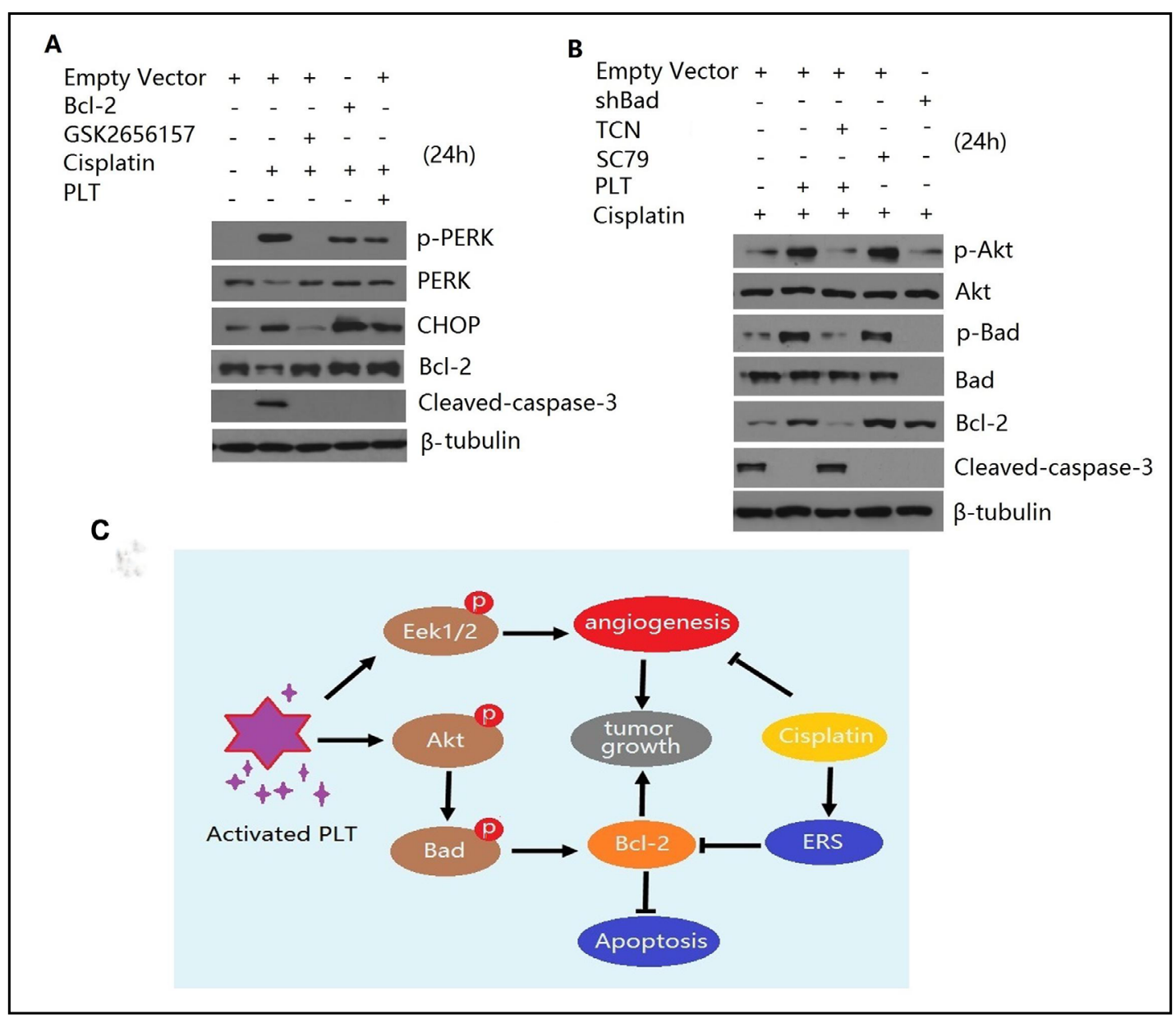

Fig. 5. PLT protected cells against cisplatin-produced apoptosis by the Akt/Bcl-2 signals. (A) Western blotting showed the expression of PERK, p-PERK, Bcl-2, cleaved-caspase-3 and CHOP in distinct groups. (B) Western blotting showed the expression of P-Bad, Bad, p-Akt, Akt, cleaved-caspase-3 and Bcl-2 in the each groups. (C) The mechanism of PLT inhibition of cisplatin-treated tumor growth showed in a brief schematic diagram.

caspase- 3 was absent. Akt is a strong inhibitory signal for apoptosis, while Bcl-2 and the caspase family activate apoptosis. Bad has been demonstrated to be a potential downstream target for Akt activation, and when activated Akt inhibits Bad (pro-apoptotic factor), Bcl-2 expression increases and caspase-3 activation is prevented [19]. Phosphorylation inactivates Bad and leads to Bcl-2 up-regulation. When A549 cells were exposed to TCN (20 $\mu \mathrm{M})$, an Akt inhibitor, together with platelets and cisplatin, the platelets induced Akt phosphorylation, Bad was inhibited, and Bcl-2 levels were down-regulated. Caspase-3 was then activated and led to cell apoptosis. When A549 cells were exposed to SC79 (25 $\mu \mathrm{M})$ Akt activator and cisplatin, they were more resistant to cisplatin, to a similar degree as that of the cells treated with cisplatin and platelets. The results suggested that Bcl-2 is an important molecule in the inhibition of apoptosis via platelets.

Finally, Bad gene was deleted in A549 cells via the retrovirus shRNA, which increased Bcl-2 levels and impeded the apoptosis created by Bcl-2 suppression. As shown in Fig. 5B, the results confirmed that the anti-apoptosis effects of platelets were mainly mediated via Akt/Bad/Bcl-2 signaling. Fig. 5C is a schematic diagram showing the mechanism underlying the effects of platelets on cisplatin-created apoptosis. 


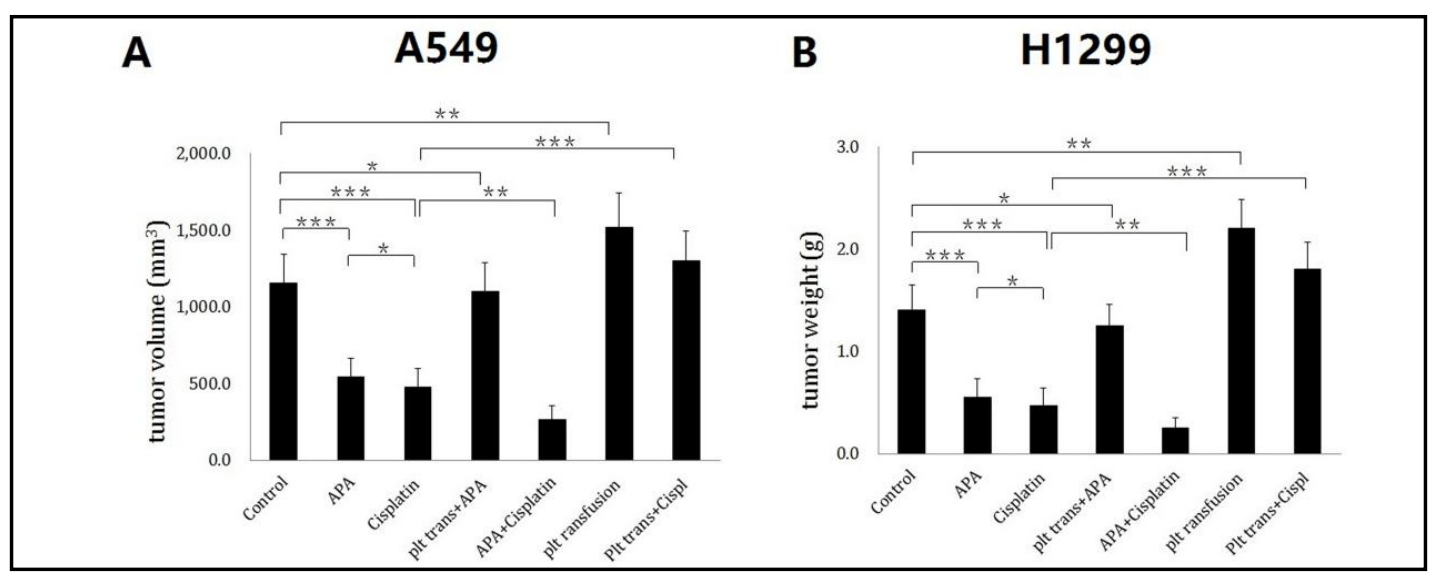

Fig. 6. Effects of platelets on response to chemotherapy and tumor growth. In vivo, A549 (A) or H1299 (B)bearing NOD/SCID mice exposure to a control IgG, APA, cisplatin, platelet transfusion, platelet transfusion + APA, APA+cisplatin, platelet transfusion+cisplatin ( $\mathrm{n}=10$ mice/group). Results are showed as mean +/-SD, $* \mathrm{p}>0.05, * * \mathrm{p}<0.05, * * * \mathrm{p}<0.01$.

Effects of thrombocytopenia and thrombocytosis on response to chemotherapy in vivo and tumor growth

To explore the effect of platelet counts on the response to platinum-based chemotherapy and tumor growth in vivo, we induced thrombocytopenia by i.v. injection with an anti-platelet antibody (CD42b/GPIb $\alpha \mathrm{mAb}, \mathrm{APA}$ ) and thrombocytosis by the transfusion of platelets three times weekly, respectively. Mice carrying A549 tumors were randomly divided into the following groups: control IgG, APA, cisplatin plus control IgG, or cisplatin plus APA, platelet transfusion, cisplatin plus platelet transfusion, and APA plus platelet transfusion. After 4 weeks, APA-treated mice had an average tumor volume that was $65 \%$ lower than the control and similar to that of cisplatin-treated mice. Thus, APA and cisplatin led to similar reductions in tumor volume. In contrast, mice treated with both cisplatin and APA had an additional reduction in average tumor volume compared with mice treated with cisplatin alone. Compared with the control, mice given APA plus a platelet transfusion showed no obvious reduction in average tumor volume, while platelet transfusions resulted in a 1.6fold increase in average tumor volume. Mice treated with platelet transfusions plus cisplatin had a 2.5-fold increase in average tumor volume compared with mice treated with cisplatin alone (Fig. 6). Similar data were gained in the H1299 model.

\section{Discussion}

In the present study, we showed that high platelet levels correlated with shorter overall survival and progression-free survival times in NSCLC patients. As a clinical end point, overall survival is affected by overlapping treatments; therefore, the clinical prognosis of thrombocytosis in association with worse overall survival indicated that platelets may confer resistance to chemotherapy. Furthermore, one of the important findings in this study was that the changes in platelet counts before and after chemotherapy in patients with NSCLC reflected the biological behavior of the tumors, suggesting that platelet counts could act as substitute biomarkers to measure chemotherapeutic response in patients with NSCLC.

In in vitro cell models, the rescue effect of platelets on cisplatin-produced apoptosis in HPMECs resulted from the activation of Akt and Erk signaling. The phosphorylation of Erk and Akt promotes angiogenesis, and Akt conveys anti-apoptotic signals [20], suggesting that Akt signaling is the key survival pathway of HPMECs. Through these two vital signaling pathways, HPMEC apoptosis was reduced and angiogenesis greatly increased. In addition, the in vivo results indicated that angiogenesis in the tissues was protected by the platelets. 
The binding of cisplatin to cell DNA and the ensuing induction of cell apoptosis are considered to be the main mechanisms of cisplatin cytotoxicity [21]. Recent research has confirmed that cisplatin-produced apoptotic signals can arise via ERS, indicating that the endoplasmic reticulum (ER) is a cytosolic target of cisplatin [22]. ERS triggers the unfolded protein reaction (UPR) and is a key response of the cell's reaction to environmental factors. UPR involves the ERS sensor PERK, ER molecular chaperone GRP78/BiP, activating transcription factor 6 (ATF6), and inositol-requiring enzyme 1, along with their downstream signals [23]. The main functions of ERS are the protection of cells under stress though the restoration of homeostasis and the reduction of damaging effects. GRP78, a calcium-binding protein mainly located in the ER, is an important regulator of this reverse process [24]. When ERS increases, the system turns to pro-apoptotic regulation to trigger cell death, even in the presence of high concentrations of GRP78. The transcription factor CHOP is the key factor in this procedure [18]. PERK-eIF2 $\alpha$-ATF4-CHOP-Bcl-2 signals are the main pathways of the proapoptotic route $[25,26]$. It has been confirmed that CHOP inhibits $\mathrm{Bcl}-2$ transcription and apoptotic cells inhibit Bcl-2 protein synthesis [27]. The Akt signaling pathway is involved in a series of cellular functions including proliferation, cell survival, angiogenesis, cell migration, and metabolism. Our data indicated that platelets activated Akt, which increased the antiapoptotic effect, prompted Bcl-2 expression, and thereby prevented caspase-3 activation under cisplatin-induced ERS. The phosphorylation of Akt and up-regulation of Bcl-2 suggested that the protective role of platelets against A549 cell apoptosis relies on the Akt-Bcl-2 signal pathway. Bad, a pro-apoptotic protein in Bcl-2-familiy, boosts cell apoptosis by preventing Bcl-2 up-regulation [28]. Western blots showed that platelet-activated Akt inactivated Bad through phosphorylation and led to an increase of Bcl-2 in A549 cells. Silenced Bad in A549 cells increased Bcl-2 expression, suggesting that the role of Bad silencing in the expression of $\mathrm{Bcl}-2$ protein was similar to that of Akt activation, which regulates Bcl-2 by Bad inactivation. Moreover, SC79 activator activated Akt, which in turn boosted Bcl-2 expression and resulted in anti-apoptosis in cisplatin-treated A549 cells. When Akt was inhibited by TCN in A549 cells exposed to cisplatin plus platelets, the platelets rescued Bcl-2 down-regulation by repressing Akt. Moreover, the expression of Bcl-2 remained the same, suggesting that anti-apoptosis activity occurred and affected the cisplatin-induced apoptotic mechanism in A549 cells. The steady expression of Bcl-2 in A549 cells was regulated by both CHOP and Akt activation. Under ERS, platelets prevented the CHOP-mediated inhibition of Bcl-2 by the Akt-Bad-Bcl-2Caspase-3 pathway, thus rescuing A549 cell apoptosis. Although our data indicated that AktBad-Bcl-2 signaling is an important pathway in platelet-created pro-survival effects, it did not remove the contribution of other anti-apoptotic signal pathways. Overall, these results demonstrated for the first time that platelets play an important cytoprotective role against cisplatin-ERS-induced apoptosis in vitro and in vivo.

The latent effects of platelet transfusion on cancer progression and survival remains uncertain. Our results further confirmed that lower platelet levels in vivo effectively reduced tumor growth, and the antitumor activity of chemotherapy was strongly neutralized by platelet transfusion. Thrombocytopenia is a common side effect of chemotherapy and responsible for increased risk of bleeding, need for reduced chemotherapy dose, and/ or delayed therapy planning in patients, which may compromise the treatment outcome [29]. The approach to the management of thrombocytopenia is mainly based on platelet transfusion. However, our results confirmed that platelet transfusion boosted tumor growth and may pose a risk to patients. Moreover, reduced platelet levels may improve the efficacy of chemotherapy [30], thus the safety parameters need to be carefully ascertained.

The plateletsused in this study wereisolated from healthy subjects. The platelet proteomes are similar in healthy individuals. The composition of the protein content of platelets reflects the current physiological state and is highly dynamic during pathophysiological processes. Moreover, the profiles of platelet proteins in patients who are either receiving or not receiving anti-cancer therapy differ from those of healthy subjects [31]. Peterson et al. evaluated and compared the levels of VEGF, bFGF, endostatin, thrombospondin-1 (TSP1), PDGF, and platelet factor 4 (PF4) in the platelets of patients with colorectal cancer and 
compared them with healthy controls. Statistically significant differences were found in the median levels of VEGF, PF4, and PDGF in the platelets of patients with cancer compared wth the healthy individuals [32]. Another recent study suggests that platelet mRNA profiles are also altered in patients with cancer, enabling discrimination between cancer patients and healthy individuals [33]. Remarkably, the present experimental protocol involved an interplay between cancer cells and platelets drawn from healthy people; thus, platelets from NSCLC patients will be used in future studies.

The potential anti-apoptosis mechanisms of platelets have been discussed in this paper. It is clear that reduced platelet levels may improve the chemotherapeutic effects. However, why do platelets have such a function and is there a molecule on the platelets that functions as the key factor? In order to identify platelet factors that may protect cancer cells from damage, Radziwon-Balicka et al. used proteomics to study the secretome of proteins released during interactions between paclitaxel-treated colonic (Caco-2) cells and platelets [6]. A number of platelet-secreted proteins are known to modulate apoptosis. These include thrombospondin-1, TGF- $\beta$, RANTES, and clusterin. Interestingly, in their model, TSP-1 significantly increased the survival of Caco- 2 cells challenged with paclitaxel [6]. We would like to investigate the presence of a molecule on the platelets that functions as the key factor using our experimental conditions.

The results presented here indicated that high platelet levels attenuated the clinical efficacy of chemotherapy and may represent a biomarker of treatment response in NSCLC patients. We also confirmed an important anti-apoptosis mechanism related to the effects of platelets and identified that platelets might confer resistance against apoptosis. Platelets increased the survival of cancer cells and permitted angiogenesis in the presence of cisplatin-a widely used chemotherapeutic drug-therefore, we forecast that reduced platelet levels or the inhibition of platelet-based anti-apoptosis in combination with chemotherapy may strengthen the efficacy of treatment in NSCLC.

\section{Clinical perspectives}

The study presented the first clinical evidence of the effect of platelet levels on therapeutic response in NSCLC. Moreover, this work revealed that platelets rescued cisplatin-induced apoptosis via the Akt/Bad/Bcl-2 signaling pathway under ERS. Reduced platelet levels or the inhibition of platelet-based pro-survival in combination with chemotherapy may strengthen the efficacy of treatment in NSCLC.

\section{Abbreviations}

NSCLC (Non-small cell lung cancer); ERS (endoplasmic reticulum stress); HPMEC (human pulmonary microvascular endothelial cells); A549 (and H1299, human lung cancer cell lines); CHOP (CCAAT/enhancer binding proteins); PERK (RNA activated protein kinase like endoplasmic reticulum kinase); ERK (extracellular regulated kinase); Bcl-2 (B-cell lymphoma-2); Bad (a member of Bcl-2 protein family); Akt (protein kinase B); bFGF (basic fibroblast growth factor); TGF- $\beta$ (transforming growth factor- $\beta$ ).

\section{Acknowledgements}

This work was supported by grants from Natural Science Foundation of Anhui Province (1608085MH229) and National Natural Science Foundation of China (30672437).

Y.-Q. Luo conceived and designed the experiments and drafted the manuscript. J.-P. Du conceived and supervised the study. Z.-F. Wang, M. Fang, J. Li and R.-N. Yang performed experiments, acquired data. Z.-F. Wang, M. Fang and R.-N. Yang analyzed the data and revised the manuscript. Y.-Q. Luo, M. Fang and Z.-F. Wang interpreted data and critically revised the manuscript. All authors read and approved the final version of the manuscript. 


\section{Cellular Physiology Cell Physiol Biochem 2018;48:2456-2469 \begin{tabular}{ll|l} 
DOI: 10.1159/000492683 & $\begin{array}{l}\text { O 2018 The Author(s). Published by S. Karger AG, Basel } \\
\text { www.karger.com/cpb }\end{array}$
\end{tabular}}

Wang et al.: Platelet Levels Affect Response to Platinum in NSCLC

\section{Disclosure Statement}

The authors declare to have no conflicts of interests.

\section{References}

1 Nurden AT: Platelets, inflammation and tissue regeneration. Thromb Haemost 2011;105:S13-33.

$>2$ Liu Y, Gao XM, Fang L, Jennings NL, Su Y, Q X, Samson AL, Kiriazis H, Wang XF, Shan L, Sturgeon SA, Medcalf RL, Jackson SP, Dart AM, Du XJ: Novel role of platelets in mediating inflammatory responses and ventricular rupture or remodeling following myocardial infarction. Arterioscler Thromb Vasc Biol 2011;31:834-841.

3 Bertozzi CC, Schmaier AA, Mericko P, Hess PR, Zou Z, Chen M, Chen CY, Xu B, Lu MM, Zhou D, Sebzda E, Santore MT, Merianos DJ, Stadtfeld M, Flake AW, Graf T, Skoda R, Maltzman JS, Koretzky GA, Kahn ML: Platelets regulate lymphatic vascular development through CLEC-2-SLP-76 signaling. Blood 2010;116:661670.

4 Erpenbeck L, Schon MP: Deadly allies: the fatal interplay between platelets and metastasizing cancer cells. Blood 2010;115:3427-3436.

5 Bambace NM, Holmes CE: The platelet contribution to cancer progression. J Thromb Haemost 2011;9:237249.

6 Radziwon-Balicka A, Medina C, O’Driscoll L, Treumann A, Bazou D, Inkielewicz-Stepniak I, Radomski A, Jow H, Radomski MW: Platelets increase survival of adenocarcinoma cells challenged with anticancer drugs: mechanisms and implications for chemoresistance. Br J Pharmacol 2012;167:787-804.

7 Palumbo JS, Talmage KE, Massari JV, La Jeunesse CM, Flick MJ, Kombrinck KW, Hu Z, Barney KA, Degen JL: Tumor cell-associated tissue factor and circulating hemostatic factors cooperate to increase metastatic potential through natural killer cell-dependent and-independent mechanisms. Blood 2007;110:133-141. Labelle M, Begum S, Hynes RO: Direct signaling between platelets and cancer cells induces an epithelialmesenchymal-like transition and promotes metastasis. Cancer Cell 2011;20:576-590.

-9 Barber EL, Boggess JF, Van Le L, Kim KH, Bae-Jump VL, Brewster WR, Soper JT, Gehrig PA: Association of Preoperative Thrombocytosis and Leukocytosis With Postoperative Morbidity and Mortality Among Patients With Ovarian Cancer. Obstet Gynecol 2015;126:1191-1197.

10 Ikeda M, Furukawa H, Imamura H, Shimizu J, Ishida H, Masutani S, Tatsuta M, Satomi T: Poor prognosis associated with thrombocytosis in patients with gastric cancer. Ann Surg Oncol 2002;9:287-291.

11 Zheng J, Cai J, Li H, Zeng K, He L, Fu H, Zhang J, Chen L, Yao J, Zhang Y, Yang Y: Neutrophil to Lymphocyte Ratio and Platelet to Lymphocyte Ratio as Prognostic Predictors for Hepatocellular Carcinoma Patients with Various Treatments: a Meta-Analysis and Systematic Review. Cell Physiol Biochem 2017;44:967-981.

$\checkmark 12$ Ohuchi M, Inoue S, Ozaki Y, Ueda K: Platelet count and mean platelet volume are associated with not only bone, soft tissue, and lymph node metastases but also with malignant pleural effusion in lung cancer patients. Neoplasma 2017;64:140-147.

13 Bottsford-Miller J, Choi HJ, Dalton HJ, Stone RL, Cho MS, Haemmerle M, Nick AM, Pradeep S, Zand B, Previs RA, Pecot CV, Crane EK, Hu W, Lutgendorf SK, Afshar-Kharghan V, Sood AK: Differential platelet levels affect response to taxane-based therapy in ovarian cancer. Clin Cancer Res 2015;21:602-610.

$>14$ Eisenhauer EA, Therasse P, Bogaerts J, Schwartz LH, Sargent D, Ford R, Dancey J, Arbuck S, Gwyther S, Mooney M, Rubinstein L, Shankar L, Dodd L, Kaplan R, Lacombe D, Verweij J: New response evaluation criteria in solid tumours: revised RECIST guideline (version 1.1). Eur J Cancer 2009;45:228-247.

15 Elrod HA, Lin YD, Yue P, Wang X, Lonial S, Khuri FR, Sun SY: The alkylphospholipid perifosine induces apoptosis of human lung cancer cells requiring inhibition of Akt and activation of the extrinsic apoptotic pathway. Mol Cancer Ther 2007;6:2029-2038.

-16 Lobov IB, Rao S, Carroll TJ, Vallance JE, Ito M, Ondr JK, Kurup S, Glass DA, Patel MS, Shu W, Morrisey EE, McMahon AP, Karsenty G, Lang RA: WNT7b mediates macrophage-induced programmed cell death in patterning of the vasculature. Nature 2005;437:417-421. 


\section{Cellular Physiology Cell Physiol Biochem 2018;48:2456-2469 \begin{tabular}{l|l|l} 
DOI: 10.1159/000492683 & $\begin{array}{l}\text { O 2018 The Author(s). Published by S. Karger AG, Basel } \\
\text { www.karger.com/cpb }\end{array}$
\end{tabular}}

Wang et al.: Platelet Levels Affect Response to Platinum in NSCLC

17 Heath-Engel HM, Chang NC, Shore GC: The endoplasmic reticulum in apoptosis and autophagy: role of the BCL-2 protein family. Oncogene 2008;27:6419-6433.

18 Oyadomari S, Mori M: Roles of CHOP/GADD153 in endoplasmic reticulum stress. Cell Death Differ 2004;11:381-389.

19 Zhu Y, Wu G, Zhu G, Ma C, Zhao H: Chronic sleep restriction induces changes in the mandibular condylar cartilage of rats: roles of Akt, Bad and Caspase-3 Int J Clin Exp Med 2014;7:2585-2592.

20 Ho L, Tan SY, Wee S, Wu Y, Tan SJ, Ramakrishna NB, Chng SC, Nama S, Szczerbinska I, Chan YS, Avery S, Tsuneyoshi N, Ng HH, Gunaratne J, Dunn NR, Reversade B: ELABELA Is an Endogenous Growth Factor that Sustains hESC Self-Renewal via the PI3K/AKT Pathway. Cell Stem Cell 2015;17:435-447.

21 Gonzalez VM, Fuertes MA, Alonso C, Perez JM: Is cisplatin-induced cell death always produced by apoptosis? Mol Pharmacol 2001;59:657-663.

-22 Mandic A, Hansson J, Linder S, Shoshan MC: Cisplatin induces endoplasmic reticulum stress and nucleusindependent apoptotic signaling. J Biol Chem 2003;278:9100-9106.

23 Gorman AM, Healy SJ, Jager R, Samali A: Stress management at the ER: regulators of ER stress-induced apoptosis. Pharmacol Ther 2012;134:306-316.

24 Lee AS: GRP78 induction in cancer: therapeutic and prognostic implications. Cancer Res 2007;67:34963499.

25 Rozpedek W, Pytel D, Mucha B, Leszczynska H, Diehl JA, Majsterek I: The Role of the PERK/eIF2alpha/ ATF4/CHOP Signaling Pathway in Tumor Progression During Endoplasmic Reticulum Stress. Curr Mol Med 2016;16:533-544.

-26 Lin JH, Li H, Yasumura D, Cohen HR, Zhang C, Panning B, Shokat KM, Lavail MM, Walter P: IRE1 signaling affects cell fate during the unfolded protein response. Science 2007;318:944-949.

27 Wang M, Kaufman RJ: Protein misfolding in the endoplasmic reticulum as a conduit to human disease. Nature 2016;529:326-335.

28 Yu F, Sugawara T, Maier CM, Hsieh LB, Chan PH: Akt/Bad signaling and motor neuron survival after spinal cord injury. Neurobiol Dis 2005;20:491-499.

29 Elting LS, Rubenstein EB, Martin CG, Kurtin D, Rodriguez S, Laiho E, Kanesan K, Cantor SB, Benjamin RS: Incidence, cost, and outcomes of bleeding and chemotherapy dose modification among solid tumor patients with chemotherapy-induced thrombocytopenia. J Clin Oncol 2001;19:1137-1146.

-30 Demers M, Ho-Tin-Noe B, Schatzberg D, Yang JJ, Wagner DD: Increased efficacy of breast cancer chemotherapy in thrombocytopenic mice. Cancer Res 2011;71:1540-1549.

31 Strohkamp S, Gemoll T, Humborg S, Hartwig S, Lehr S, Freitag-Wolf S, Becker S, Franzen B, Pries R, Wollenberg B, Roblick UJ, Bruch HP, Keck T, Auer G, Habermann JK: Protein levels of clusterin and glutathione synthetase in platelets allow for early detection of colorectal cancer. Cell Mol Life Sci 2018;75:323-334.

-32 Peterson JE, Zurakowski D, Italiano JE, Jr., Michel LV, Connors S, Oenick M, D’Amato RJ, Klement GL, Folkman J: VEGF, PF4 and PDGF are elevated in platelets of colorectal cancer patients. Angiogenesis 2012;15:265273.

33 Best MG, Sol N, Kooi I, Tannous J, Westerman BA, Rustenburg F, Schellen P, Verschueren H, Post E, Koster J, Ylstra B, Ameziane N, Dorsman J, Smit EF, Verheul HM, Noske DP, Reijneveld JC, Nilsson RJA, Tannous BA, Wesseling P, et al.: RNA-Seq of Tumor-Educated Platelets Enables Blood-Based Pan-Cancer, Multiclass, and Molecular Pathway Cancer Diagnostics. Cancer Cell 2015;28:666-676. 